\title{
An unusual presentation of chronic lymphocytic leukemia
}

Dinesh Atwal, Mihir Raval, Belal Firwana, Jeanette Ramos, Appalanaidu Sasapu

Department of Hematology Oncology, University of Arkansas for Medical Sciences, Winthrop Rockefeller Cancer Institute, Little Rock, Arkansas, USA

\begin{tabular}{|c|}
\hline Access this article online \\
\hline Website: www.avicennajmed.com \\
\hline DOI: 10.4103/ajm.AJM_171_16 \\
\hline Quick Response Code: \\
\hline
\end{tabular}

\section{ABSTRACT}

Chronic lymphocytic leukemia/small lymphocytic lymphoma (CLL/SLL) is a B-cell lymphocytic neoplasm with indolent clinical course. If identified early, observation is opted. Many variables lead to the initiation of treatment. Authors describe a 62-year-old male presenting with shortness of breath and found to have white cell count of $1360 \times 10^{9} / \mathrm{L}$ and subsequently was diagnosed with CLL/SLL. The patient received leukapheresis along with tumor lysis treatment and systemic chemotherapy with fludarabine, cyclophosphamide, and rituximab regimen. His course was complicated with deep venous thrombosis, extensive cutaneous, and sinus mucosa involvement by CLL/SLL. The patient clinically improved and on follow-up clinic visits documented normalization of his white cell counts. The case report brings up a rare presentation of CLL/SLL with such an extreme high white cell count, leukostasis symptoms and extramedullary involvement of disease and encourages providers to be vigilant of rare presentation of CLL/SLL.

Key words: Chronic lymphocytic leukemia, hyperleukocytosis, leukostasis

\section{INTRODUCTION}

Chronic lymphocytic leukemia/small lymphocytic lymphoma (CLL/SLL) is a neoplasm of functionally incompetent mature B-cell lymphocytes. It is usually indolent in nature and found incidentally. CLL/SLL is the most prevalent type of leukemia in western world adults accounting for around $25 \%-35 \%$ of Leukemia in the United States. ${ }^{[1]}$ At presentation, $25 \%-50 \%$ of the patients are asymptomatic. Lymphadenopathy is the most common presentation. Splenomegaly, hepatomegaly, pallor, and petechiae are less common signs and symptoms of CLL/SLL. One of the diagnostic criteria for CLL/SLL is the presence of absolute lymphocyte count (ALC) greater than $5 \times 10^{9} / \mathrm{L},{ }^{[2]}$ and it is usual to see ALCs as high as $100 \times 10^{9} / \mathrm{L}$. It is exceedingly rare to find extreme hyperleukocytosis with white blood cell $(\mathrm{WBC})$ count greater than $1000 \times 10^{9} / \mathrm{L}$ with only a few case reports in the literature. ${ }^{[3,4]}$ We describe a patient with CLL/SLL who presented with a WBC count of $1360 \times 10^{9} / \mathrm{L}$ and developed cardiorespiratory failure.

Address for correspondence: Dr. Appalanaidu Sasapu, University of Arkansas for Medical Sciences, Winthrop Rockefeller Cancer Institute, 4301 West Markham Street, \#508, Little Rock, Arkansas, USA.

E-mail: asasapu@uams.edu
The patient received one session of leukapheresis, which resolved the symptoms. The patient also developed skin rash, determined to be cutaneous involvement by CLL/SLL, and leukemic infiltration of sinus mucosa during the illness. Leukemic involvement of the skin by CLL/SLL is not as common as other leukemias/lymphomas, ${ }^{[5]}$ and leukemic infiltration of mucosa is a rare phenomenon. ${ }^{[6]}$

\section{CASE REPORT}

A 62-year-old healthy male patient was transferred to our hospital with shortness of breath progressively worsening over few months. He was found to have extreme hyperleukocytosis with WBC count of $1360 \times 10^{9} / \mathrm{L}$, essentially $100 \%$ lymphocytes. His laboratory findings showed hemoglobin of $5.2 \mathrm{~g} / \mathrm{dL}$, and concern for tumor lysis with potassium $6.4 \mathrm{mEq} / \mathrm{L}$, uric acid $8.0 \mathrm{mg} / \mathrm{dL}$, phosphate $4.6 \mathrm{mg} / \mathrm{dL}$, and

This is an open access article distributed under the terms of the Creative Commons Attribution-NonCommercial-ShareAlike 3.0 License, which allows others to remix, tweak, and build upon the work non-commercially, as long as the author is credited and the new creations are licensed under the identical terms.

For reprints contact: reprints@medknow.com

Cite this article as: Atwal D, Raval M, Firwana B, Ramos J, Sasapu A. An unusual presentation of chronic lymphocytic leukemia. Avicenna J Med 2017;7:133-6. 
creatinine $1.4 \mathrm{mg} / \mathrm{dL}$. The peripheral blood smear showed a predominance of small, mature lymphocytes with a subset of intermediate to large cells, comprising approximately $15 \%$ of lymphocytes. A subset of these larger cells had morphology consistent with prolymphocytes [Figure 1a]. Flow cytometry showed a kappa-restricted B-cell population, comprising 95\% of analyzed events that co-expressed CD19, CD20, $\mathrm{CD} 5, \mathrm{CD} 23$, and CD200 that were negative for FMC7, CD38, and CD10; this immunophenotype is consistent with CLL/SLL; by forward scatter, a subset of cells are larger (approximately, $13 \%$ of analyzed events) with the same immunophenotype. Computed tomography scan showed diffuse lymphadenopathy in mediastinum and abdomen.

The patient was treated with intravenous fluid hydration, rasburicase, allopurinol, and also received hydroxyurea for cytoreduction and packed red blood cell transfusion. Later on, the patient developed cardiorespiratory failure requiring intubation. A chest X-ray showed perihilar and bibasilar pulmonary opacities as well as a pleural effusion consistent with congestive heart failure likely secondary to leukostasis. A subsequent transthoracic echocardiogram (TTE) showed an ejection fraction (EF) of $30 \%$. Due to worsening shortness of breath, the patient received a single session

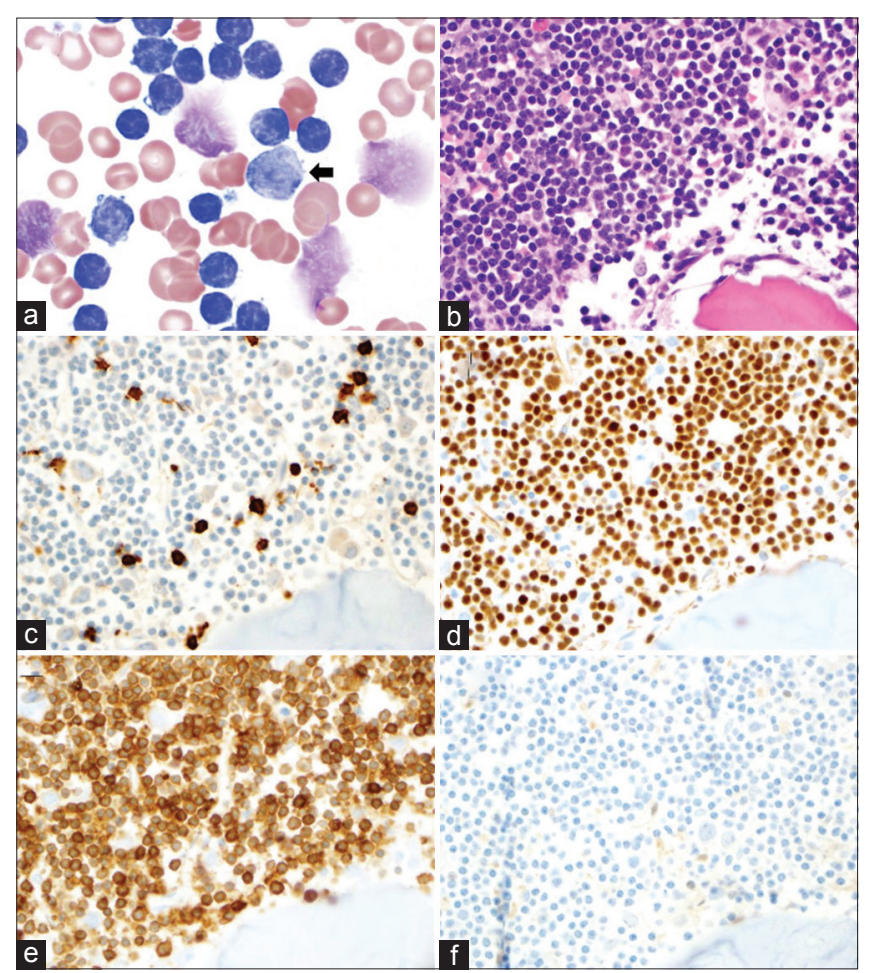

Figure 1: (a) Peripheral smear $(\times 1000)$ demonstrating a predominance of small, mature lymphocytes, scattered smudge cells, and a rare large cell with morphology consistent with a prolymphocyte (arrow); (b) Bone marrow biopsy $(\times 400)$ demonstrating sheets of small mature lymphocytes; (c) CD3 stains a few scattered, background T-cells $(\times 400)$; (d) PAX-5 stains the majority of small lymphocytes ( $\times 400)$; (e) PAX-5-positive B-cells co-express CD5 ( $\times 400)$; (f) B-cells are negative for BCL-1 $(\times 400)$ of leukapheresis, which decreased his WBC count to $757 \times 10^{9} / \mathrm{L}$, and his clinical status improved leading to extubation in few days. Repeat TTE showed an improved EF of $40 \%-45 \%$. The patient underwent bone marrow biopsy that showed a hypercellular bone marrow ( $>95 \%$ cellular) with a diffuse infiltrate of small mature lymphocytes without diagnostic morphologic evidence of large cell transformation [Figure 1b-f]. Erythroid and granulocyte lineages were depressed and left-shifted. Fluorescent in situ hybridization analysis showed $13 \mathrm{q}$ deletion. The patient also had Zap-70 expression with an unmutated immunoglobulin heavy chain variable gene.

During hospital course, the patient developed an erythematous maculopapular rash over the upper back and chest [Figure 2]; a subsequent skin biopsy showed dense inflammatory infiltrate comprising of small lymphocytes, histiocytes, and occasional eosinophils consistent with cutaneous involvement by CLL/SLL [Figure 3a-c]. The patient subsequently developed dysphonia and a sore throat that did not respond to antibiotics. The biopsy of nasal mucosa showed an atypical lymphoid infiltrate mostly B-cells co-expressing CD5, consistent with leukemic infiltration by CLL/SLL [Figure 3d-f]. His course of illness was also complicated by the development of deep venous thrombosis in internal jugular (IJ) vein mostly related to catheter and he was started on rivaroxaban. At the time of initiation of chemotherapy, his WBC count was $590 \times 10^{9} / \mathrm{L}$. The patient was started on chemotherapy with fludarabine, cyclophosphamide, and rituximab (FCR), and with two cycles of FCR, the complete blood count normalized, and his cutaneous involvement resolved.

\section{DISCUSSION}

CLL/SLL is indolent leukemia that can remain asymptomatic for years. CLL/SLL rarely presents with hyperleukocytosis $\left(\mathrm{WBC}\right.$ count $\left.>100 \times 10^{9} / \mathrm{L}\right)$. Leukostasis, defined as a pathophysiological process where large aggregates of leukocytes impede the blood flow in the microcirculation causing end organ damage usually resulting in cardiopulmonary and neurological symptoms, is even less common in CLL/SLL associated hyperleukocytosis. ${ }^{[7]}$ The

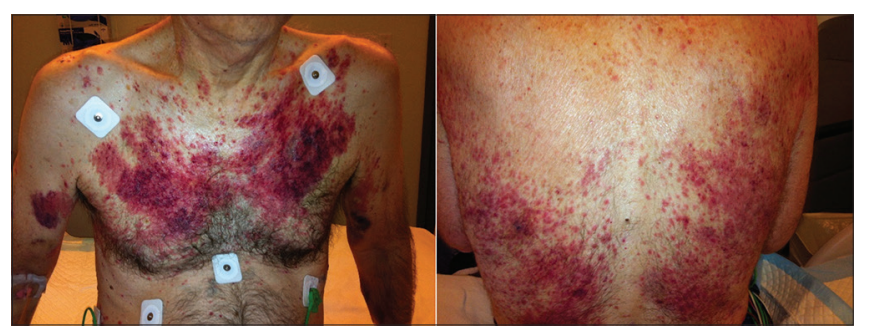

Figure 2: Maculopapular rash on patient's chest and back 


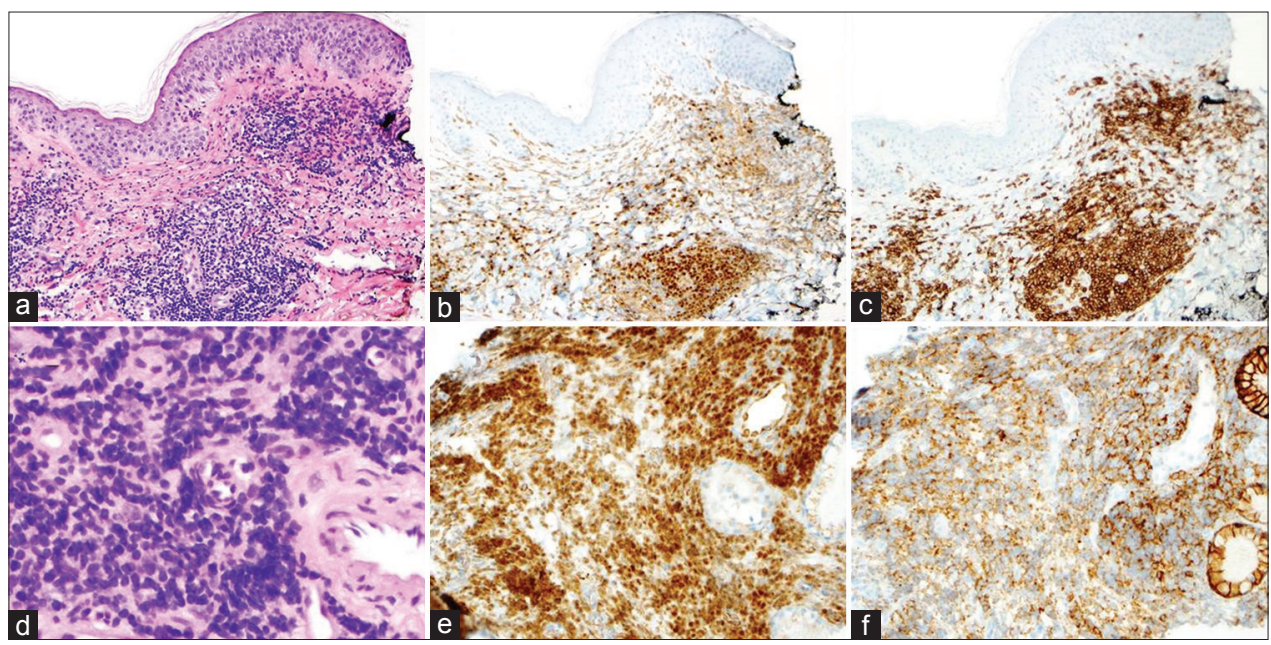

Figure 3: (a) $\mathrm{H}$ and $\mathrm{E}$ stain from the upper back skin biopsy demonstrates a dense infiltrate of small, mature lymphocytes in the superficial dermis ( $\times 100)$; (b) Pax- 5 stains increased and aggregates of B-cells ( $\times 100$ ); (c) CD5 stains B-cells and intermixed T-cells ( $\times 100)$; (d) $\mathrm{H}$ and E demonstrates a diffuse infiltrate of small, mature B-cells involving the nasal mucosa ( $\times 400)$; (e) Pax-5 demonstrates that the majority of lymphocytes are B-cells ( $\times 200)$; (f) These B-cells co-express CD5

classical presentation of leukostasis is typically described in acute leukemias, particularly acute myeloid leukemias. It is a rare in CLL/SLL because of the small size of mature lymphocytes and their inherent characteristics. ${ }^{[7,8]}$

Our patient presented with a WBC count of $1360 \times 10^{9} / \mathrm{L}$ associated with cardiorespiratory failure, which was a sequela of leukostasis, and leukapheresis aided in the resolution of the symptoms. CLL/SLL presenting with such a high WBC count is an exceedingly rare phenomenon. Nevertheless, there are few case reports on CLL/SLL associated with extreme hyperleukocytosis with WBC count $>1000 \times 10^{9} / \mathrm{L} .^{[3,4]}$ The range of WBC count among these studies is $1125 \times 10^{9} / \mathrm{L}$ to $2000 \times 10^{9} / \mathrm{L}$. All of these patients developed symptoms secondary to leukostasis, treated with leukapheresis to decrease WBC load with symptom resolution.

The patient described in our report also developed thrombosis of IJ vein that required treatment with rivaroxaban. Only one of the previous cases with extreme hyperleukocytosis (WBC count $>1000 \times 10^{9} / \mathrm{L}$ ) developed venous thromboembolism (VTE) in the form of common femoral vein thrombosis. ${ }^{[3]}$ There have been few case reports on patients with CLL/SLL presenting with VTE. ${ }^{[3,9]}$ Whittle et al. found an incidence of $1.45 \%$ per patient year in CLL/SLL, which is higher compared to the general population but less than lymphoma patients $(0.15 \%-0.2 \%$ in general population). ${ }^{[9]}$

Another interesting aspect of presentation in our patient was leukemic infiltration of skin and nasal mucosa and vocal cord. Although cutaneous lesions can be seen in patients with leukemias/lymphomas (3\%-50\%), this finding is less common in CLL/SLL. ${ }^{[5]}$ When present, it typically presents with erythematous macules, papules, plaques or nodules, ${ }^{[5,10]}$ and our patient developed an erythematous maculopapular rash over back and chest. Moreover, it is extremely rare to find leukemic infiltration of mucosa, as seen in this case, with only a few reports of leukemic infiltration of mucosa of oropharyngeal cavity, paranasal sinuses, and nasolacrimal duct, ${ }^{[6]}$ reported in the literature. Melton and Pearlman concluded that mucosal infiltration of oropharynx or paranasal sinuses is found in $<1 \%$ of CLL cases. ${ }^{[6]}$

\section{CONCLUSION}

Extreme hyperleukocytosis is exceedingly rare in patients with CLL/SLL. Hyperviscosity and leukostasis are rare but potential complications seen in these patients, warranting leukoreduction. Since chemotherapy takes few days to reduce the WBC count, it is prudent to perform leukapheresis, which can reduce the counts rapidly and can be life-saving in leukostasis. CLL/SLL patients with hyperleukocytosis can also present with leukemic infiltration of the skin and mucosa. This infiltration by leukemic cells is not necessarily correlated with the stage of CLL/SLL and may, in fact, be dependent on the tumor burden or the WBC count.

\section{Financial support and sponsorship}

Nil.

\section{Conflicts of interest}

There are no conflicts of interest.

\section{REFERENCES}

1. Siegel RL, Miller KD, Jemal A. Cancer statistics, 2016. CA Cancer J Clin 2016;66:7-30. 
2. Hallek M, Cheson BD, Catovsky D, Caligaris-Cappio F, Dighiero G, Döhner $\mathrm{H}$, et al. Guidelines for the diagnosis and treatment of chronic lymphocytic leukemia: A report from the International Workshop on Chronic Lymphocytic Leukemia updating the National Cancer Institute-Working Group 1996 guidelines. Blood 2008;111:5446-56.

3. Cukierman T, Gatt ME, Libster D, Goldschmidt N, Matzner Y. Chronic lymphocytic leukemia presenting with extreme hyperleukocytosis and thrombosis of the common femoral vein. Leuk Lymphoma 2002;43:1865-8.

4. Durzynski T, Konopka L, Traczyk Z. Leukostasis syndrome in a case of chronic lymphocytic leukemia. Pol Merkur Lekarski 1999;6:30-1.

5. Robak E, Robak T. Skin lesions in chronic lymphocytic leukemia. Leuk Lymphoma 2007;48:855-65.
6. Melton MF, Pearlman AN. Chronic lymphocytic leukemia of the oropharyngeal cavity and paranasal sinuses: A case series and literature review. Int Forum Allergy Rhinol 2015;5:1055-8.

7. Lichtman MA, Rowe JM. Hyperleukocytic leukemias: Rheological, clinical, and therapeutic considerations. Blood 1982;60:279-83.

8. Porcu P, Cripe LD, Ng EW, Bhatia S, Danielson CM, Orazi A, et al. Hyperleukocytic leukemias and leukostasis: A review of pathophysiology, clinical presentation and management. Leuk Lymphoma 2000;39:1-18.

9. Whittle AM, Allsup DJ, Bailey JR. Chronic lymphocytic leukaemia is a risk factor for venous thromboembolism. Leuk Res 2011;35:419-21.

10. Rosman IS, Nunley KS, Lu D. Leukemia cutis in B-cell chronic lymphocytic leukemia presenting as an episodic papulovesicular eruption. Dermatol Online J 2011;17:7. 